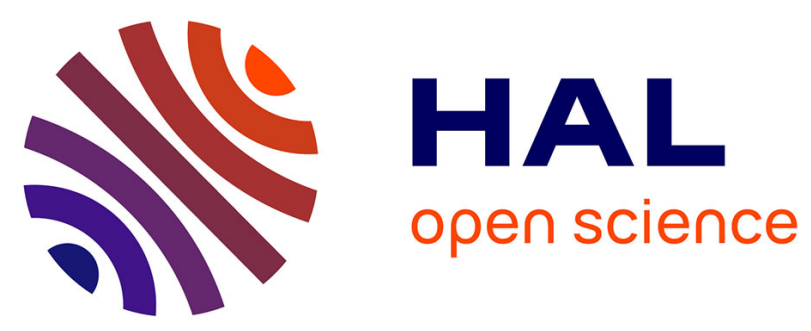

\title{
On the vortex parameter estimation using wide band signals in active acoustic system
}

Angela Digulescu, Teodor Petrut, Ion Candel, Florentina Bunea, Georgiana

Dunca, Diana Bucur, Cornel Ioana, Alexandru Serbanescu

\section{- To cite this version:}

Angela Digulescu, Teodor Petrut, Ion Candel, Florentina Bunea, Georgiana Dunca, et al.. On the vortex parameter estimation using wide band signals in active acoustic system. OCEANS 2014 OCEANS '14 MTS/IEEE. Ocean Regeneration, Apr 2014, Taipei, Taiwan. pp.978-1-4799-3646-5/14, 10.1109/OCEANS-TAIPEI.2014.6964552 . hal-00985763

\section{HAL Id: hal-00985763 https://hal.science/hal-00985763}

Submitted on 30 Apr 2014

HAL is a multi-disciplinary open access archive for the deposit and dissemination of scientific research documents, whether they are published or not. The documents may come from teaching and research institutions in France or abroad, or from public or private research centers.
L'archive ouverte pluridisciplinaire HAL, est destinée au dépôt et à la diffusion de documents scientifiques de niveau recherche, publiés ou non, émanant des établissements d'enseignement et de recherche français ou étrangers, des laboratoires publics ou privés. 


\title{
On the vortex parameter estimation using wide band signals in active acoustic system
}

\author{
Angela Digulescu ${ }^{1,2, *}$, Teodor Petrut ${ }^{1}$, Ion Candel $^{1}$, Florentina Bunea ${ }^{3}$, Georgiana Dunca ${ }^{4}$, Diana Bucur ${ }^{4}$, \\ Cornel Ioana ${ }^{1}$ and Alexandru Serbanescu ${ }^{2,5}$ \\ ${ }^{1}$ Grenoble Institute of Technology, GIPSA-lab, Saint Martin D'Heres, France \\ ${ }^{2}$ Military Technical Academy, Faculty of Military Electronic and Information Systems, Bucharest, Romania \\ ${ }^{3}$ National Institute for R\&D in Electrical Engineering ICPE-CA, Bucharest, Romania \\ ${ }^{4}$ Power Engineering Faculty, University "Politehnica" Bucharest, Romania \\ 5"Lumina" - The University of South East Europe, Bucharest, Romania \\ Email: adigulescu@mta.ro
}

\begin{abstract}
This paper proposes a new method for detection of the vortex presence in fluid flow based on an active acoustic system. The experiment that validates the theory was done on a reduced scale facility using ultrasonic transceivers. The main objective was to highlight the effect of a cavitation vortex on an applied wide band signal. In order to accomplish that, the Recurrence Plot Analysis (RPA) was investigated which emhasizes similar states of a dynamic process. The Tests were done from no vortex cavitation flow to vortex cavitation flow and backward.
\end{abstract}

Index Terms- non-intrusive monitoring, RPA, phasespace, recurrence matrix, diagonal lines.

\section{INTRODUCTION}

Analysis of vortex dynamics is an important operation in a large number of applications such as turbine monitoring, detection and localization of underwater objects, characterization of natural phenomena, etc. The effect of the vortex existance can be quantified in terms of temporal pressure variation and also in frequency variations (Doppler effect).

This paper proposes an acoustic approach of detecting the appearence and presence of a vortex in a closed hydraulic test loop.

The objective of the work is to emphasize the effect of the vortex upon the recorded signal in order to identify the different phases of evolution of the phenomenon.

An experiment was set up by increasing, then, by decreasing the water velocity in a pipeline. The non-intrusive monitoring techique used a wide-band signal impulse continuously emitted and recorded during the entire process. The RPA method was applied on the recorded signals in order to characterize the state of the flow in its evolution. Further the signals were processed and studied from their recurrences frequencies point of view.

This paper is organised as follows: in section 2, it is presented the relation between the vortex flow and vortex spectrum. The point of depart in this research was that the received Doppler spectrum is unambiguosly related with the spatial velocity distribution of a Rankine vortex [1]. The RPA method is described in section 3 and, then, the quantification of the diagonal lines specific for the recurrence matrix is highlighted.
The spectal analysis of the diagonal lines presents a new and interesting spectral approach which will be also shown. In section 4 , the experiment and the results are presented and discussed. The conclusions and perspectives are highlighted in section 5.

\section{THE VORTEX FLOW AND VORTEX SPECTRUM}

An acoustic wave interacting with a vortex presents a Doppler spectrum signature on its spectral magnitude.

The flow of an incompressible fluid of velocity $\mathbf{u}(\mathbf{r}, t)$ is characterized by the Navier-Stokes equation, (1). At the same time, the flow is fully characterized by adding the mass continuity equation (2). These equations may be formulated in terms of vorticity vector $\omega$, and giving the suitable initial and boundary conditions, a desired vortical structure can be mathematically modeled to facilitate the determination of the vortical solutions describing any vortex model. We recall the two relations metioned above [1], [2]:

$$
\begin{gathered}
\rho \cdot\left(\frac{\partial \mathbf{u}}{\partial t}+\mathbf{u} \cdot \nabla \mathbf{u}\right)=\rho \frac{\partial \mathbf{u}}{\partial t}+\rho \omega \times \mathbf{u}+\frac{1}{2} \nabla \rho \mathbf{u}^{2}=-\nabla p-\mu \nabla \times \omega \\
\nabla \cdot \mathbf{u}=\nabla \cdot(\nabla \times \omega)=0
\end{gathered}
$$

where $p$ is the fluid pressure, $\rho$ is the fluid density, $\mathbf{u}$ is the flow velocity vector and $\nabla$ is the del operator.

It is supposed that the vortex is a swirling flow field with closed streamlines. Also, the Rankine vortex model has a tangential velocity $v(r)$ which increases linearely with the radius $r$ in the forced vortex region of radius $r_{\max }$, and decreases in the free vortex region. Therefore, the polar coordinates velocities are [3]:

$$
u(r)=a ; w(r)=0 ; v(r)=\left\{\begin{array}{c}
\frac{r}{r_{\max }} v_{\max }, r \leq r_{\max } \\
(\text { forced vortex region }) \\
\frac{r_{\max }}{r} v_{\max }, r>r_{\max } \\
(\text { freevortex region })
\end{array}\right.
$$


where the velocities $u, w, v$ are the axial, radial and, respectively, tangential components defined in a cylindrical coordinate system for the fluid velocity $u$.

Physically, such type of vortex has an inner circular region of rigid-body rotation, being entirely defined by a vorticity field, and an outer region which is vorticity-free, the speed being inversely proportional to the distance from the origin.

The analytical solution 3 of the equations (1) and (2) reflect directly the frequency signature of the vortex through the solution of $\omega$. Therefore, it follows [1]:

$$
u(r)=a ; w(r)=0 ; v(r)=\left\{\begin{array}{c}
\omega \cdot r, r \leq r_{\max } \\
(\text { forced vortex region }) \\
\frac{\omega r_{\max }^{2}}{r}, r>r_{\max } \\
(\text { freevortex region })
\end{array}\right.
$$

More details about the spectral magnitude of a Doppler velocity bin can be found in [3].

The purpose of the work is to design the signal processing approach that will provide information about the vortex's parameters.

\section{ReCURREnCE Plot AnAlysis}

In this section, the signal analysis is approached from the perspective of nonlinear times series analysis that characterizes the general dynamic system (such in the applicative context).

The recurrence plot analysis has its first origins in the nonlinear time series analysis which begins with a time series obtained from a mesured set of data:

$$
\mathbf{x}=\{x[1], x[2], \ldots, x[N]\}
$$

Then the time series is represented in a $m$-dimension space, named the phasespace. The trajectory represented in the phase space is given by the corresponding vectorial representation:

$$
\overrightarrow{v_{i}}=\sum_{k=1}^{m} x[i+(k-1) d] \cdot \overrightarrow{e_{k}}, i=\overline{1, M}
$$

where $x[\cdot]$ is the sample from the time series (equation (4)), $\overrightarrow{e_{k}}$ are the unit vectors of the axis of the phasespace, $M=N-$ $(m-1) d, d$ is the delay parameter and $m$ is the dimension of the phasespace, called the embedding dimension. The choice of these two parameters is very important and will be detailed.

Once the trajectory is obtained, the RPA method computes the distance/ recurrence matrix [4]. This matrix is obtained using a certain type of distance between two points $i$ and $j$ on the trajectory. For the recurrence matrix, these distances are compared with a threshold:

$$
\begin{gathered}
D\left(\overrightarrow{v_{i}}, \overrightarrow{v_{j}}\right)=\mathcal{D}\left(\overrightarrow{v_{i}}, \overrightarrow{v_{j}}\right) \\
R_{i, j}=\Theta\left(\varepsilon(i)-D\left(\overrightarrow{v_{i}}, \overrightarrow{v_{j}}\right)\right)
\end{gathered}
$$

where $D\left(\overrightarrow{v_{i}}, \overrightarrow{v_{j}}\right)$ is the element of the distance matrix corresponding to the points $i$ and $j, \mathcal{D}(\cdot, \cdot)$ is the type of distance applied on the trajectory points (Euclidean distance, norm L1, norm infinity, angular distance, etc.), $R_{i, j}$ is the element of the recurrence matrix, $\varepsilon$ is the threshold which

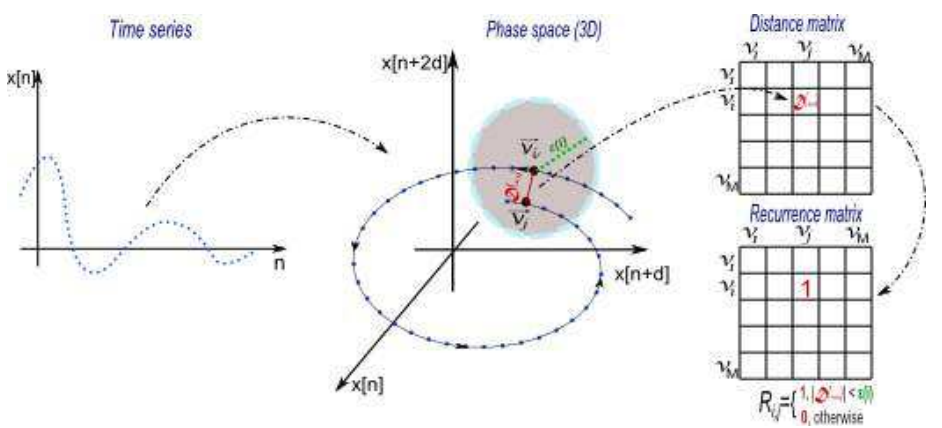

Figure 1: The RPA method illustration using the Euclidean distance

determines whether or not two points $i$ and $j$ are recurrent and $\Theta$ is the Heaviside step function. Generally, $\varepsilon$ is constant.

The distance matrix is represented through a continous colored scale instead, the recurrence matrix contains just $\{0,1\}$ values colored in black and white (see figure 1). According to the application, each matrix can be exploited.

When choosing the delay parameter, $d$, one must pay attention to the quality of the trajectory [5], which is directly influenced by it. If the chosen delay is too small, the trajectory is overfolded around the first bisector of the phasespace, therefore the trajectory is redundant. Moreover, if the delay is too large, the trajectory could appear much too complicated, therefore being irrelevant.

Hereby, for the proper choice of the delay, it must be taken into consideration the statistical indepedence property between the points of the time series. The most used criteria is the mutual information [5]:

$$
I_{A B}\left(a_{i}, b_{j}\right)=\log _{2}\left(\frac{P_{A B}\left(a_{i}, b_{j}\right)}{P_{A}\left(a_{i}\right) P_{B}\left(b_{j}\right)}\right)
$$

where $P_{A}(a)$ is the probability of getting the $a$ value from measuring $A$ system and $P_{A B}(a, b)$ is the probability of getting at the same time the $a$ value from measuring $A$ system, respectively the $b$ value from meauring the $B$ system. The first local minumum was considered to be the optimal choice.

For the optimal value of the embedding dimension, $m$, it must be considered that if the embdedding dimension is smaller than the real dimension of representation, then this trajectory would be a projection of the real trajectory which would include points closer to each other than on the real trajectory. But, if the embedding dimension is greater than the real one, then the computation resources and time would increase significantly.

Therefore, the most popular method used is the False Nearest Neighbor (FNN) [4], [5], [6]. The optimal value of the embdedding dimension is the first value of FNN equal to zero.

The next step is to quantify the diagonal lines on the distance matrix. Hereby, this quantification, also presented in [7], will be computed as follows:

$$
s d[n]=\frac{1}{M-n} \sum_{i=1}^{M-n} D\left(v_{i}, v_{i+n}\right)
$$


where $M$ is presented below equation (5), $n=\overline{0, M-1}$ and $D\left(v_{i}, v_{i+n}\right)$ are the elements of the $n^{t h}$ diagonal line of the distance matrix (the 0 diagonal corresponds to the main diagonal).

Afterwards, the Fourier transform of the diagonal lines quantification is applied:

$$
S D[k]=\sum_{n=0}^{M-1} s d[n] \cdot e^{-i 2 \pi k n / M}
$$

The major advantage of this method is that it highlights several extra frequency components than the usual Fourier transform. To confirm this affirmation and to illustrate the mathematical operations without losing the generality, the time series considered is:

$$
x[n]=\cos (\Omega n) \cdot \cos (\omega n), \Omega \gg \omega
$$

The time series from equation (10) highlights in the simplest way possible the interaction between the acoustic wave of $\Omega$ frequency and a rotational vortex of $\omega$ frequency.

To simplify the calculus, it is suppossed that the time series is represented in $2 D$, therefore the embedding dimension $m=2$, and the distance applied on the distance matrix is the squared Euclidean distance. So, it results that:

$$
v_{i}=x[i] \cdot \overrightarrow{e_{1}}+x[i+d] \cdot \overrightarrow{e_{2}}
$$

$D\left(v_{i}, v_{i+n}\right)=(x[i]-x[i+n])^{2}+(x[i+d]-x[i+d+n])^{2}$ $=E_{i}^{2}(n)+E_{i+d}^{2}(n)$

Using equation (10) in equation (12) leads to:

$$
E_{i}(n)=2 \alpha \cdot \cos \frac{\Omega(2 i+n)}{2} \cos \frac{\omega(2 i+n)}{2}
$$

where $\alpha=\cos \frac{(\Omega+\omega) n}{2} \cong \cos \frac{(\Omega-\omega) n}{2}$ considering that $\Omega \gg$ $\omega$.

Replacing the result from equation (13) in equation (12), it becomes:

$$
\begin{aligned}
& D\left(v_{i}, v_{i+n}\right)= \\
& =(2 \alpha)^{2}\left[\frac{1}{4}(\cos [\Omega(2 i+n)]-1)(\cos [\omega(2 i+n)]-1)+\right. \\
& \left.+\frac{1}{4}(\cos [\Omega(2 i+2 d+n)]-1)(\cos [\omega(2 i+2 d+n)]-1)\right]
\end{aligned}
$$

Therefore, the quantification of the diagonal lines highlights:

$$
\begin{aligned}
s d[n] \sim & D\left(v_{i}, v_{i+n}\right)= \\
& =\alpha^{2}\left(\cos \left[2 \Omega\left(i+\frac{n}{2}\right)\right] \cos \left[2 \omega\left(i+\frac{n}{2}\right)\right]-\right. \\
& -\cos \left[2 \Omega\left(i+\frac{n}{2}\right)\right]-\cos \left[2 \omega\left(i+\frac{n}{2}\right)\right]+1 \\
& +\cos \left[2 \Omega\left(i+d+\frac{n}{2}\right)\right] \cos \left[2 \omega\left(i+d+\frac{n}{2}\right)\right]- \\
& \left.-\cos \left[2 \Omega\left(i+d+\frac{n}{2}\right)\right]-\cos \left[2 \omega\left(i+d+\frac{n}{2}\right)\right]+1\right)
\end{aligned}
$$

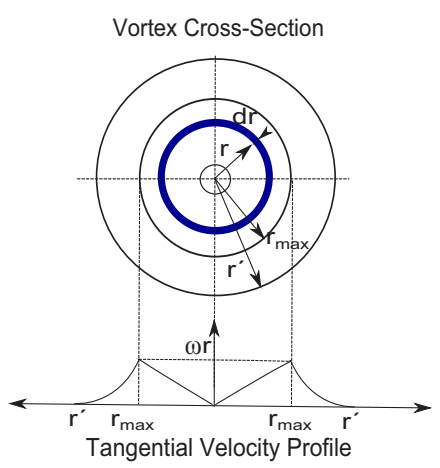

(a)

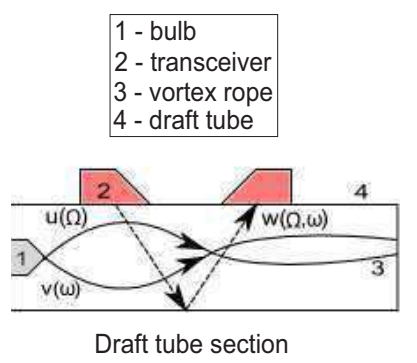

(b)
Figure 2: The transversal (a) and longitudinal (b) representation of small scaled draft tube used for experiments

From the equation 15, it can be observed that on the spectral representation of the diagonal lines quantification two extra components appear, besides the classical components $\Omega \pm \omega$ : one component at $2 \Omega$ and the other at $2 \omega$.

Considering the fact that the effect of the vortex can be attributed to the $2 \omega$ spectral component, the experimental part will aim to explore this characteristic in order to emphasize the evolution of the vortex.

\section{THE EXPERIMENT AND RESULTS}

For the experimental part, there were used two transceivers positioned in "V" configuration as shown in figure 2.

The transceivers which work at $1 \mathrm{MHz}$ central frequency emitted a wide band signal with the same central frequency. The signal $u(\Omega)$ send from the emitter has a cubic characteristic law.

A closed hydraulic test loop is used to simulate the dynamic of a rotational vortex rope existing in hydraulic turbines. A stator is placed in a divergent section to create a vortex rope, similar with vortex ropes often met in hydropower plant turbines The vortex is characterized by an unknown law $v(\omega)$. Hereby, at the receiver, the signal carries the interaction between the emitted signal and the studied vortex in $w(\Omega, \omega)$. The purpose of the experiment is to highlight the signature of the vortex from the received signal.

The wide band signals emitted were continuosly recorded and their frequency content was chosen to fit the flow and the vortex in order to retreive from the signals the apparition, development and damping of the vortex. During the experiment, the water flow was varied from 4 to $19 \frac{\text { liter }}{\mathrm{sec}}$ and backwards, in order to determine the critical flow rates for which the vortex appears/dampens. Two data sets were obtained corresponding to the two flow configurations (low flow $->$ high flow and high flow $->$ low flow).

The experimental configuration is presented in figure 3 .

A comparison between the classical approach of the FFT on the received signal and its $S D$ version is presented in figure 4. It must be mentiond that both signals (the actual received signal and its corresponding RPA diagonal lines quantification $(S D F F T)$ ) had zero mean before the FFT computation. 


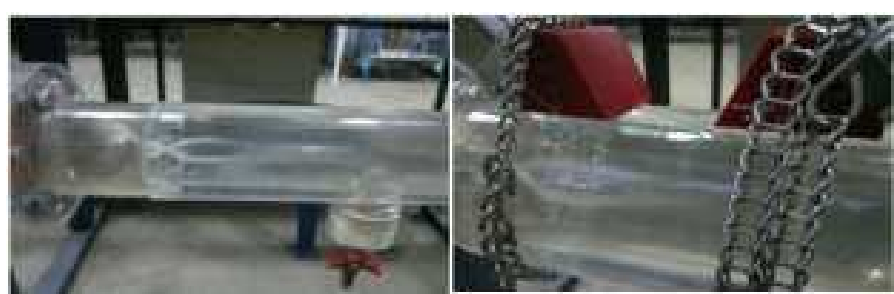

(a)

(b)

Figure 3: The vortex and the experimental "V" configuration of the ultrasonic transceivers
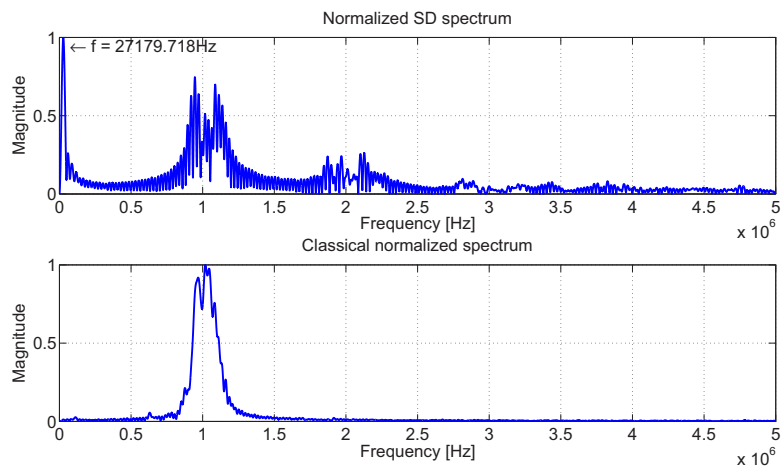

Figure 4: The spectral representation of the received signal in terms in terms of $S D$ and classical approach

While the classical FFT approach presents a wide-band signal centered on $1 \mathrm{MHz}$, the $S D F F T$ approach points out the spectral components mentioned in equation (15). The $\Omega \pm \omega$ component is similar to the classical FFT aproach (making the discrimination of the vortex effect hard to realize (because $\Omega \gg \omega)$ ). The $2 \Omega$ component resembles the $\Omega \pm \omega$ component. In addition, the $2 \omega$ spectral component is also present on the representation.

Therefore, using this propriety of the SDFFT, the evolution of the vortex during the flow rate increase or decrease is studied. The maximum of $2 \omega$ spectral component has been determined and represented for each measurement. The results are shown in figure 5.

The results obtained for the two sets of signals (down-up and up-down) present a shift of the $2 \omega$ component position. This position varies, but it has a trend that follows the vortex intensity: increases when the vortex intensity increases, decreases while it decreases, respectively.

Moreover, it can be seen that while there is no vortex (steady flow - the beginning of the increasing part, the end of the decreasing part) the position of this component is almost unchanged. When the vortex appears - no cavitation flow to cavitation flow, the deviation of the position is higher, but it follows the water flow trend.

The main cause of the deviation is the instability that the vortex brings into the system. Still, the singularities present in the cavitation flow part may appear because the parameters of the RPA method ( $m$ and $d$ ) are not well suited for the received signal or the signal may be corrupted.

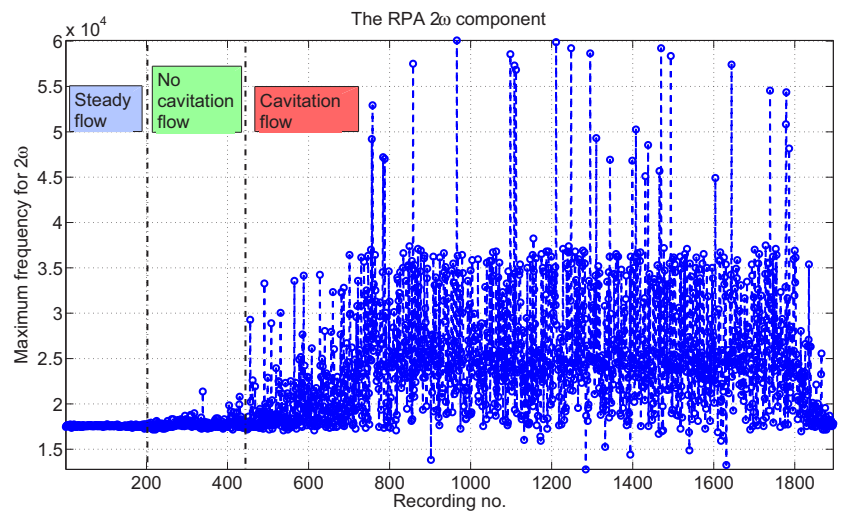

(a)

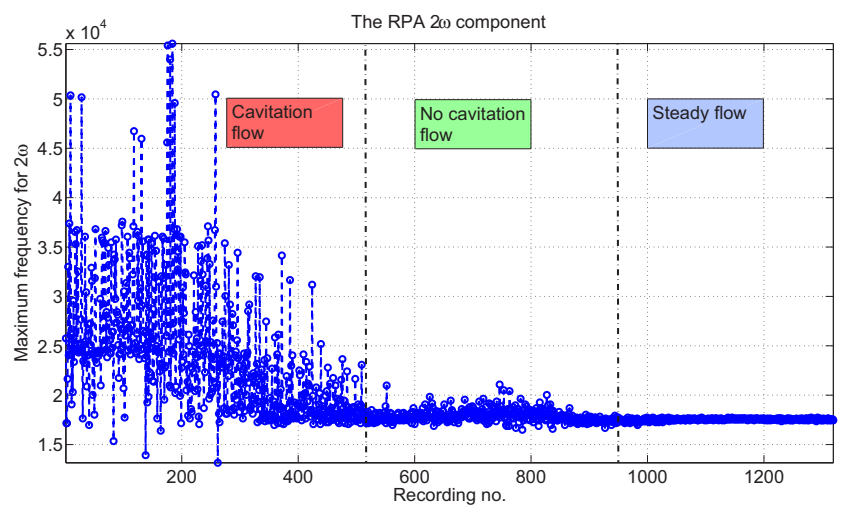

(b)

Figure 5: The RPA $2 \omega$ spectral component while increasing (a) and decreasing (b) the water flow from 4 to $19 \frac{l}{s}$ and backwards

In conclusion, the evolution of the flow can be characterized by this signal processing approach.

\section{Conclusions}

This paper proposes to find a new tool capable to estimate the parameters of a vortex in a draft tube being generated by a Kaplan or Francis runner.

The experiment was done by varying the flow rate from $4 \frac{l}{s}$, without vortex to a flow rate of $19 \frac{l}{s}$, where the vortex is clear eye visible, as presented in figure 3 .

In this paper was presented a new method - RPA, specific for chaotic systems, and, in order to detect the vortex signature, the spectral analysis of the diagonal lines quantification was proposed.

In [5], it is highlighted that the diagonal lines reflect the fundamental frequency of a signal. Moving forward, in this paper it was shown that the spectral analysis of their quantification brings into light the effect of a interaction between two different waves, in certain conditions.

The theory was confirmed by the experimental tests where it could be seen that the spectral component of the vortex is present in the diagonal lines quantification analysis. This component describes the evolution of flow and of vortex. 
Future work considers to confirm the new approach on other applications.

\section{ACKNOWLEDGEMENT}

This work was supported in part by the "Smart Hydro Monitoring Project" labeled by the "Tenerdis and Minalogic Competitivity Pole".

\section{REFERENCES}

[1] N. Takahashi, T. Miyazaki, "The Influence Of Turbulence On A Columnar Vortex With Axial Flow", Proceedings in Applied Mathematics and Mechanics 6, 2006.

[2] K. A. Emanuel, "A note on the stability of columnar vortices", Journal of Fluid Mechanics, vol. 145, 1984.

[3] W. L. Rubin, "Radar-Acoustic Detection of Aircraft Wake Vortices", Journal of Atmospheric and Oceanic Technology, vol. 17, 2000.

[4] N. Marwan, S. Schinkel, J. Kurts, "Recurrence plots 25 years later Gaining confidence in dynamic transitions", Europhysics Letters, 101, 20007, 2013.

[5] A. Serbanescu, O. Stanasila, F.-M. Birleanu, "Analiza neliniara a seriilor de timp", Military Technical Academy Publishing, Bucharest, 2011

[6] M. Small, "Applied nonlinear time series analysis. Applications in phisics, physiology and finance", World Scientific Publishing, New Jersey, 2005.

[7] Florin-Marian Birleanu, Ion Candel, Cornel Ioana, Cedric Gervaise, Alexandru Serbanescu, Gheorghe Serban, "A vector approach to transient signal processing”, ISSPA 2012, Montreal, Canada, July 3-5 2012. 